\title{
Anti-Adhesive And Antiproliferative Synergistic Surface Modification Of Intraocular Lens For Reduced Posterior Capsular Opacification
}

This article was published in the following Dove Press journal: International Journal of Nanomedicine

Yuemei Han, (D) ${ }^{1, *}$ Junmei Tang, ', J Jiayi Xia,' Rui Wang,' Chen Qin,' Sihao Liu,' Xia Zhao,' Hao Chen, ${ }^{1,2}$ Quankui Lin (D) ${ }^{1,2}$

'School of Ophthalmology \& Optometry, Eye Hospital, Wenzhou Medical University,

Wenzhou 325027, People's Republic of China;

${ }^{2}$ Wenzhou Institute of Biomaterials and

Engineering, Chinese Academy of Sciences,

Wenzhou 32500, People's Republic of China

*These authors contributed equally to this work

\section{Video abstract}

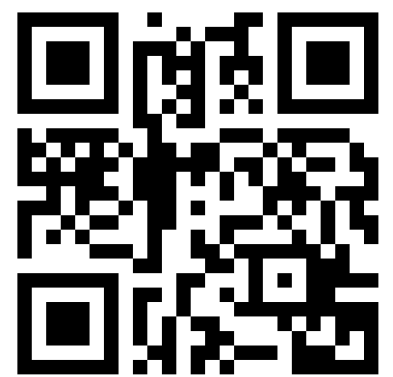

Point your SmartPhone at the code above. If you have QR code reader the video abstract will appear. Or use: https://youtu.be/selJKG6NMZk

Correspondence: Hao Chen

School of Ophthalmology \& Optometry, Eye Hospital, Wenzhou Medical University, 270

Xueyuan Xi Road, Wenzhou 325027, People's Republic of China

$\mathrm{Tel} / \mathrm{Fax}+8657788067962$

Email chenhao823@mail.eye.ac.cn

Quankui Lin

School of Ophthalmology \& Optometry, Eye Hospital, Wenzhou Medical University, 270

Xueyuan Xi Road, Wenzhou 325027, People's

Republic of China

Tel/Fax +8657788067962

Email linqk@wmu.edu.cn
Background: Posterior capsular opacification (PCO) is the main complication after intraocular lens (IOL) implantation in cataract surgery, which is the result of lens epithelial cell (LEC) adhesion, proliferation and migration on the IOL and at the lens capsule interface. Hydrophilic surface modification, such as surface heparinization, decreases the cell adhesion, which has been commercialized and used clinically. However, clinical long-term observation results show no significant difference between the pristine and heparinized IOLs.

Methods: To prevent PCO over the long time span, we modified the IOLs with an antiproliferative drug-loaded hydrophilic coating. The antiproliferative drug doxorubicin (DOX)-incorporated chitosan (CHI) nanoparticle was fabricated by sodium tripolyphosphate (TPP) gelation. Such antiproliferative drug-loaded CHI-TPP-DOX nanoparticles (CTDNP) were used as one of the building blocks to prepare polyelectrolyte multilayer with heparin (HEP) via layer-by-layer assembly, obtaining (HEP/CTDNP $)_{n}$ multilayers. The assembly process was characterized by quartz crystal microbalance with dissipation (QCM-D). The drug release behavior of the coating was investigated by ultra-HPLC (UPLC). In vitro cell experiments were carried out to monitor the effects of multifunctional coatings on cellular adhesion, proliferation and migration. And the intraocular implantation was performed on rabbits to evaluate the in vivo PCO inhibitory effect of such surface-functionalized IOLs.

Results: The positively charged CTDNP was successfully prepared by ionic gelation. The QCMD results indicate the successful preparation of the (HEP/CTDNP $)_{n}$ multilayer film. Drug release profiles showed that surface-multifunctionalized IOL had drug-sustained release properties. In vitro cell culture results showed significant inhibition of adhesion, proliferation and migration of LECs after surface modification. The in vivo results showed that the IOLs with multifunctionalized surface can effectively reduce the posterior hyperplasia and Soemmering's ring (SR) formation.

Conclusion: These findings suggested that such multifunctionalized drug-eluting IOLs can effectively reduce the posterior hyperplasia and SR formation when intraocular implantation has a major impact on reducing PCO incidence. Thus they have a great potential in improving patient vision recovery and maintenance.

Keywords: surface modification, intraocular lens, posterior capsular opacification, drugeluting coating, nanoparticle

\section{Introduction}

Cataract remains the leading cause of blindness worldwide despite the availability of effective surgery in developed countries. It is reported that about $47.8 \%$ blindnesses are caused by cataract, and recently, there are about 20 million cataractous blindness worldwide. ${ }^{1}$ Cataract is the crystalline lens opacification, which results in 
light obstruction and gradual vision loss. The first line of treatment for cataract is to replace the opaque lens with an intraocular lens (IOL) in the capsular bag. ${ }^{1}$ The cataract surgery recovers the vision effectively. However, a common postoperative complication, namely posterior capsular opacification (PCO), occurs in high incidence. ${ }^{2}$ It is reported that the PCO incidence is around $20-40 \%$ in adults' cases after IOL implantation for 5 years, whereas the incidence is as high as $100 \%$ in children's cases. ${ }^{2}$ PCO is an abnormal tissue formed by the residual lens epithelial cells (LECs), which may be due to the wound healing reactions after cataract-IOL surgery. ${ }^{3}$ The only way to treat PCO in clinic is neodymium-doped yttrium aluminum garnet (Nd:YAG) laser capsulotomy. ${ }^{2}$ However, the laser irradiation requires additional costs and is accompanied by several complications, such as retinal detachment, high intraocular pressure and so on.

Till date, many attempts have been taken to reduce the incidence of PCO. Antiproliferative drugs were used by researchers to test if these drugs could prevent residual LECs' proliferation. For example, they injected drugs into the lens capsule during IOL implantation surgery, or soaked IOL in drug solution before implantation. ${ }^{4,5}$ With disadvantages of burst drug release and accompanied side effects to the adjacent tissues, these actions did not remarkably reduce the PCO incidence either. ${ }^{5}$ Surface modification provides an alternative way to improve the biocompatibility of biomaterials. ${ }^{6,7}$ Scientists have designed bio-inert or hydrophilic surface coatings on IOL surface, such as surface immobilization with $\mathrm{CF}_{4}$, hydrophilic HEP, polyethylene glycol, phosphorylcholine moieties and so on. ${ }^{8-11}$ So far, the surface-heparinized IOL has been used clinically and serves as one of the high-end IOL types. ${ }^{12}$ These surface coatings decrease cell adhesion to prevent PCO. However, the obtained in vivo results were not as good as expected. Current clinical investigations also showed that there was no significant difference in PCO incidence between the pristine and heparinized IOLs in the long run. ${ }^{12}$ In previous studies, we have generated enhanced anti-adhesive surface modifications to improve the IOL biocompatibility via surface-initiated-reversible addition-fragmentation chain transferpolymerization and natural polyelectrolyte layer-by-layer (LbL) deposition method. ${ }^{13-17}$ The enhanced hydrophilic surface modification decreased the posterior capsular hyperplasia, whereas they also could not eliminate the PCO incidence thoroughly. ${ }^{13-16}$ The sole anti-adhesion modification is likely to be inadequate in actual applications.
In this study, the design of antiproliferative drug-sustained release was introduced into the hydrophilic surface coatings on IOL for postoperatively long-term PCO prevention. Based on the excellent anti-adhesive to the cell of the natural polysaccharide multilayers investigated previously, ${ }^{13}$ the drug-loaded nanoparticles were prepared and used as one of the building blocks in the multilayered surface coating fabrication. Assuming that the antiproliferative drug-eluting will induce the residual cell apoptosis in the capsule, the PCO inhibition may be greatly improved in such multifunctionalized IOLs. The doxorubicin (DOX) was used as the model antiproliferative drug in this research. It was incorporated into the chitosan (CHI) nanoparticles via electrostatic condensation between the cationic CHI and ionic sodium tripolyphosphate (TPP), obtaining CHI-TPP-DOX nanoparticles (CTDNP). The positively charged nanoparticles were then deposited with negatively charged polysaccharide HEP on IOL surface via LbL technique, forming HEP/CTDNP polyelectrolyte multilayer surface coating (Figure 1). The feasibility of the coating onto IOL as well as the in vitro cell behavior and intraocular PCO inhibition were investigated. It is hypothesized that the PCO inhibition may be greatly improved on the antiproliferative drug-loaded polysaccharide multilayer-modified IOLs.

\section{Materials And Methods Materials And Reagents}

Chitosan (CHI, molecular weight (MW) around 179.17 kDa, high viscosity (>400 mPa.s)), TPP and HEP (185 USP units/ $\mathrm{mg}$ ) were purchased from Aladdin Biochemical Technology Co., Ltd. (Shanghai, China). Doxorubicin hydrochloride (DOX) was purchased from Meilun Biotechnology Co., Ltd. (Dalian, China). Polyethyleneimine (PEI, $\mathrm{MW}=25,000), \quad N$-(3-dimethylaminopropyl)- $N$ '-ethylcarbodiimide hydrochloride (EDC), $N$ - hydroxysulfosuccinimide sodium (NHS) and fluorescein diacetate (FDA) were purchased from Sigma-Aldrich (St Louis, MO, USA). FBS, DMEM/F12 (1:1) cell culture media, 0.05\% Trypsin-EDTA and other cell culture-related reagents were purchased from Invitrogen (Waltham, MA, USA). Human LEC line (HLE B3, CRL-11421 ${ }^{\mathrm{TM}}$ ) was originated from American Type Culture Collection. Foldable hydrophobic IOLs were supplied by 66 Vision Tech Co., LTD (Suzhou, China). The clinical postoperative administration drugs were obtained from Eye Hospital of Wenzhou Medical University 


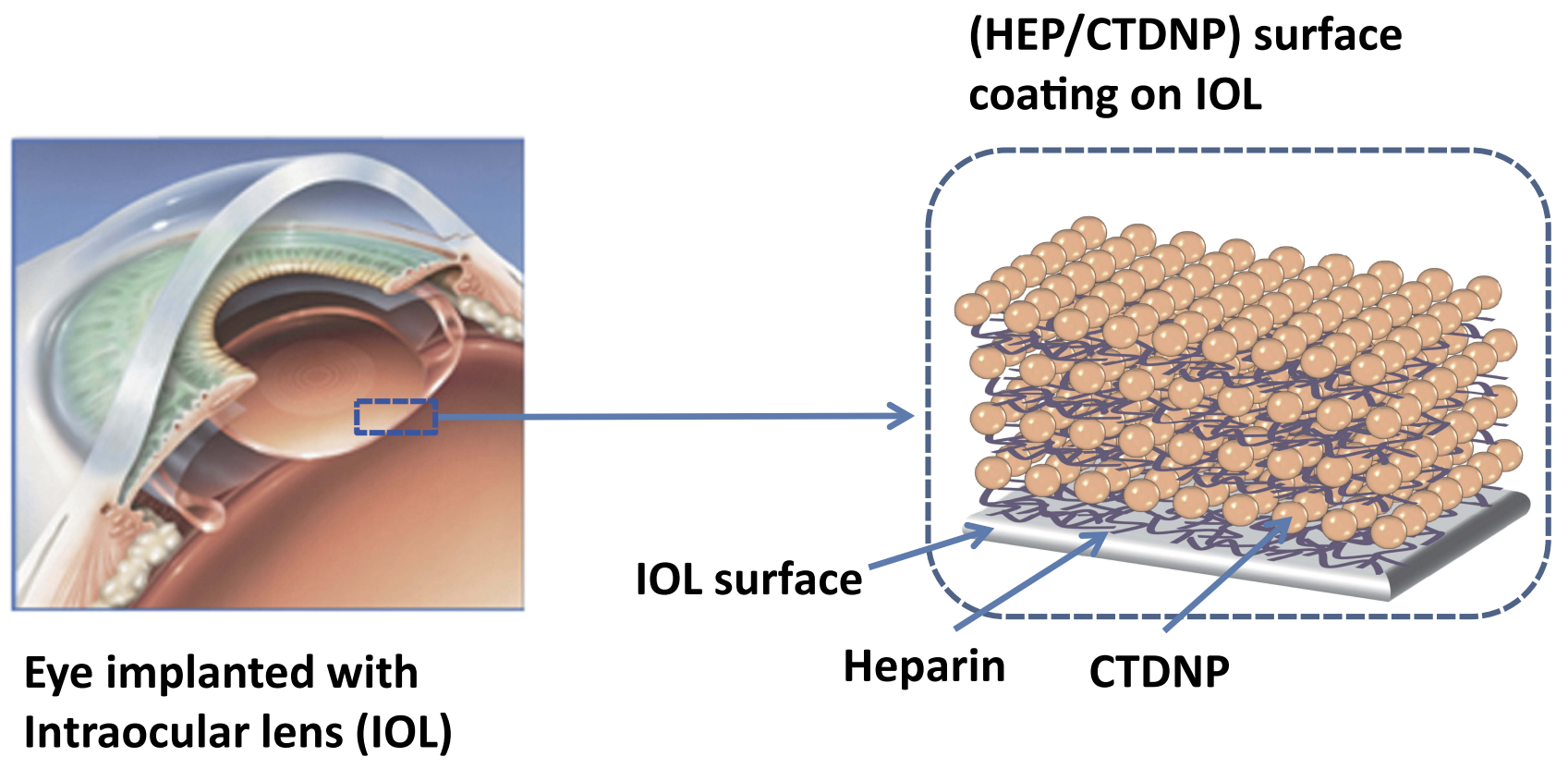

Figure I Schematic illustration of drug-eluting hydrophilic coating-modified IOL fabricated via layer-by-layer deposition of heparin and drug-loaded chitosan nanoparticles.

(Wenzhou, China). All other chemicals were analytical grade and used without further purification.

\section{$\mathrm{CHI}$ Nanoparticles Fabrication And Characterization}

The CHI nanoparticles were prepared by the polyelectrolyte condensation between TPP and CHI. ${ }^{18}$ Briefly, 0.2\% (w/v) TPP aqueous solution was added dropwise into $0.1 \% \mathrm{CHI}$ solution (w/v in $1 \%$ acetic acid) under magnetic stirring $(500 \mathrm{rpm})$ at room temperature, and incubated for another 30 mins. A series of nitogen/ phosphorus (N/P) ratios were carried out to optimize the CHI-TPP nanoparticles, where the N/P ratio refers to the molar ratio of nitrogen element in the repeating unit of $\mathrm{CHI}$ and phosphorus element in TPP. The DOX-loaded nanoparticles (CHI-TPP-DOX nanoparticle, CTDNP) were prepared under the optimized conditions with a similar protocol, in which the CHI solution was supplemented with $0.05 \%(\mathrm{w} / \mathrm{v})$ DOX. The drug-loaded nanoparticles were further chemically cross-linked via $40 \mathrm{mM}$ EDC and $20 \mathrm{mM}$ NHS at room temperature for $1 \mathrm{hr}$, and then used for multilayer construction. The particle size and zeta potential of the nanoparticles were determined by dynamic light scattering with Autosizer 3000 (Malvern Instruments Limited, UK). Each sample was measured thrice to obtain the Z-average of particle size and the average zeta potential. The morphology of the nanoparticle $(\mathrm{N} / \mathrm{P}=3)$ was observed by scanning electron microscope (SEM, Phenom-World, the Netherlands).

\section{Construction And Characterization Of The HEP/CTDNP Multilayer}

Polyester IOL materials and hydrophobic acrylic IOLs were used as the substrates for polyelectrolyte multilayer fabrication. The fabrication procedure was similar with our previous publications. ${ }^{13,17,19,20}$ Each experiment was initiated by PEI treatment for $24 \mathrm{hrs}$, which resulted in a positively charged substrate surface. Then, the substrates were dipped in $1 \mathrm{mg} / \mathrm{mL}$ HEP solution for 15 mins. After that, it was rinsed by acetic buffer solution and dried by a stream of $\mathrm{N}_{2}$. The HEP-terminated substrates were subsequently dipped into the freshly prepared CTDNP solution for 15 mins. The same rinsing and drying processes were carried out after CTDNP deposition. The alternating HEP and CTDNP deposition cycles were continued until determined bilayers of HEP/CTDNP were fabricated.

The growth of the HEP/CTDNP multilayer was followed by quartz crystal microbalance with dissipation (QCM-D, Q-sense AB, Sweden). ${ }^{13,21}$ Briefly, the quartz crystal was excited at its fundamental frequency $(\sim 5 \mathrm{MHz}$, $\mathrm{v}=1$ ), as well as at the 3rd, 5th, 7th, 9th and 11th overtones $(\mathrm{v}=3,5,7,9,11$ corresponding to $15,25,35,45$ and 55 $\mathrm{MHz}$, respectively). Changes in resonance frequencies $(\Delta F)$ and relaxation $(\Delta D)$ of the vibration once the excitation was stopped were recorded. In detail, the clean quartz 
crystal was installed in the QCM chamber and the acetic buffer was injected at equilibrium. Then, the PEI solution was continuously injected into the chamber at $100 \mu \mathrm{L} / \mathrm{min}$ until equilibrium, followed by the buffer pumping at the same speed. Then, the HEP and CTDNP solutions were alternatively injected until equilibrium and separated by the washing solution. After $\mathrm{n}$ cycles of HEP and CTDNP injection, the $(\mathrm{HEP} / \mathrm{CTDNP})_{\mathrm{n}}$ multilayers were obtained. The curves of frequency shift vs time and dissipation vs time were recorded, respectively. The transmittances of the surface-coated IOLs were carried out by the UV-visible spectrophotometer (UV-Vis, UV3100, Thermo Fisher, USA). The optical images of surface-coated IOLs were observed by stereomicroscope (Digital Sight DS-U3, Nikon, Minato-ku, Tokyo, Japan).

\section{In Vitro Drug Release Study}

The drug release behaviors of the multilayer films were investigated. ${ }^{19,22}$ The IOL materials were incubated in a 4 $\mathrm{mL}$ releasing buffer at $37^{\circ} \mathrm{C}$ with gentle shaking. PBS $(\mathrm{pH}=7.4)$ and acetate buffer $(\mathrm{pH}=5.5)$ were used for monitoring the physiologicaland pathological conditions, respectively. At certain time points, $1 \mathrm{~mL}$ release buffer was collected and stored in the refrigerator while another 1 $\mathrm{mL}$ fresh buffer was added into the incubation solution. After all the samples were collected, the buffers were filtrated with $0.22 \mu \mathrm{m}$ cellulose nitrate membrane and tested by the ultra-HPLC (UPLC) with UPLC BEH C18 columns (Waters, USA) at room temperature. The detect method is according to Pharmacopoeia of the People's Republic of China. A series of DOX standard solution with concentrations from 0.01 to $5 \mu \mathrm{g} / \mathrm{mL}$ were prepared to obtain the standard curve. The mobile phase was a mixture of buffer solution (sodium lauryl sulfate $1.44 \mathrm{~g}$ and $0.68 \mathrm{~mL}$ of phosphoric acid dissolved in $500 \mathrm{~mL}$ of deionized water)/acetonitrile/methanol (500/500/60, v/v/ v). The flow rate was $0.3 \mathrm{~mL} / \mathrm{min}$. The sample quantity was $0.5 \mathrm{~mL}$ and the detection wavelength was $254 \mathrm{~nm}$. The concentration was obtained via the UPLC analysis software according to the standard curve.

\section{In Vitro LECs Test}

For cell adhesion test, IOL materials with a diameter of $6 \mathrm{~mm}$ were prepared and sterilized by UV irradiation, and placed in the 96-well cell culture plates. LECs were digested by $0.25 \%$ trypsin, centrifugalized and resuspended in DMEM/F12 containing 10\% FBS after 95\% cell confluence. The cell seeding density was 1000 or
2000 cells/well depending on different experiments. The effects of surface modification on anti-adhesion and antiproliferation of LECs were assessed by FDA staining after culture for 24 hrs. Fluorescent images were acquired by fluorescent microscopy (Nikon) with fluorescein filter (488 $\mathrm{nm} /$ excitation). The cell number was counted using the ImageJ software adding with artificial judgment. To monitor the cell migration and proliferation in lens capsule, cells were seeded on the peripheral parts of the cell culture wells. After cell attachment for $24 \mathrm{hrs}$, the IOL materials were placed in the central areas and the cells were cultured for another $48 \mathrm{hrs}$. The unmodified materials and the no drug-loaded surface coating materials were used as controls. The cells were stained by H\&E and optical images were taken.

\section{In Vivo Intraocular Implantation And Evaluation}

The animal experiment was approved by local laboratory animal committee (Laboratory Animal Ethics Committee of Wenzhou Medical University with approved institutional protocols set by the China Association of Laboratory Animal Care). Eight-weeks-old male rabbits (obtained from the Wenzhou Medical University Laboratory Animal Center, Approval No. 2016-0057) were used for the animal experiment ( $\mathrm{n}=10,2$ groups), following Laboratory animals - guideline for ethical review of animal welfare (2018, China). IOLs' implantation surgical procedure and postoperative evaluation were the same as our previous investigations. ${ }^{13,16}$ Briefly, after making a $2.2 \mathrm{~mm}$ temporal corneal incision, the anterior chamber was filled with ophthalmic viscosurgical device (OVD) and a continuous curvilinear capsulorhexis (CCC) was subsequently created to allow $360^{\circ} \mathrm{CCC}-\mathrm{IOL}$ overlap after IOL implantation. After hydrodissection and phacoemulsification, the folded IOLs were implanted into the capsular bag with a dedicated IOL injector. Then, the OVD was completely removed from IOL boundary, capsular bag and anterior chamber. According to the clinical cataract surgeries, subconjunctival injection of triamcinolone acetonide was carried out to reduce postoperative inflammation. Other postoperative treatments, including levofloxacin and tobramycin-dexamethasone eye drops, were carried out four times daily for 2 weeks, and two times for another 2 weeks. Regular slit lamp microscopic observation was carried out to judge postoperative acute inflammation and the long-term PCO development. All 
rabbits were euthanized by air embolism after excessive anesthesia in the 10th week. The eyeballs were collected and sectioned at the equator. Gross examination of the anterior segment was performed from the posterior aspect (Miyake-Apple View) to assess the severity of the capsular bag opacification. The severity of central PCO (CPCO, the capsule areas behind IOL optical center with a diameter of $3.0 \mathrm{~mm}$ ), peripheral PCO (PPCO, the capsule areas behind the periphery of the IOL optic, except CPCP area) and Soemmering's ring (SR) formation ( equatorial region of the capsular bag, outside the IOL optic area) were scored from grades 0 to $3 .^{13}$ After observation, the lens capsules with IOL inside were separated and the stereomicroscopic images were taken. Some of the eyeballs were fixed for histochemical staining and observation. Briefly, the lens capsules were fixed in $4 \%$ paraformaldehyde, dehydrated in $30 \%$ sucrose solution. Then, the tissues were made into frozen section and followed with H\&E staining. The optical images were taken to observe the ocular tissue morphology and the posterior capsule hyperplasia thickness, which was used to judge the PCO degree as well.

\section{Statistical Analysis}

All experiments were conducted in triplicate, and data were expressed as mean $\pm \mathrm{SD}$. An independent sample $t$-test was used for data analyses in PCO scores, oneway ANOVA was conducted for the statistical analysis of cell adhesion ratios and cell apoptosis ratios with different multilayers modification. Statistically significant difference was defined at a confidence level of $95 \%(* P<0.05)$.

\section{Results And Discussion}

Almost all the IOL material investigations for PCO prevention focused on the surface hydrophilic modification. ${ }^{9,23-27}$ The aim of these surface hydrophilic modifications was to decrease the residual LEC adhesion after IOL implantation. The surface-heparinized IOL is the only surface-modified IOL approved for clinical use so far. However, recent clinical research has demonstrated that there is no significant difference in PCO incidences between the patients who were treated by the HEP-modified IOLs or the pristine IOLs implantation. ${ }^{12}$ In our previous animal investigations, the surface hydrophilic or superhydrophilic modifications had some positive effects on the PCO prevention in short time observations. ${ }^{13-17,28}$ However, if the postoperative observation time duration prolonged, the PCO also started to develop in the hydrophilic-modified IOLs. ${ }^{14-16}$ In a normal crystalline lens, lens epithelium monolayer is confined to the anterior capsular surface and ends at the equatorial lens bow. A single layer of flat cuboidal epithelial cells, "A" cells as called, with minimal mitotic activity corresponds to the anterior lens capsule zone. Anterior epithelial cells in the equator form the equatorial lens bow. Unlike " $A$ " cell layer, these cells have mitotic activity and undergo multiplication. New lens fibers are continuously produced in this zone throughout life. ${ }^{29}$ Although the cataract surgery techniques have been greatly improved in recent years, the LECs still cannot be removed completely during the operation. The residual LEC adhesion, proliferation, migration and fibrosis result in the PCO after the surgery. $^{3}$ The surface with hydrophilic modification only resists residual LECs adhering onto the IOL surfaces. ${ }^{13-16}$ However, as the cells are located in the relatively closed lens capsular bags, the cells may proliferate from capsular base membrane to the IOL surface in the long run. That is, the anti-adhesive surface modification only delays the PCO incidence, but cannot prevent its appearance. So the antiproliferative drug administration to inhibit cell proliferation is necessary. Drug-eluting surface coating suggests a promising way for localized drug delivery of the implantable materials. ${ }^{6,19,30}$ The surface modification of drug-eluting coating is an effective way to prevent the postoperative complications of the implants, such as cardiovascular stent. The antiproliferative drugs incorporated coatings serve as depots and sustained released drugs, which inhibit the cell proliferation and reduce the restenosis effectively in the long run. In the present investigation, the drug-eluting coating design was introduced into the IOL surface modification, aiming to reduce PCO after its implantation. The antiproliferative drug-loaded $\mathrm{CHI}$ nanoparticles were prepared and immobilized onto the IOL surface via LbL method.

\section{Drug-Loaded CHI Nanoparticle Preparation}

Among all the natural polymers, $\mathrm{CHI}$ is one of the most promising commercialized biopolymers. It is a linear polysaccharide composed of 2-acetamido-2-deoxy- $\beta$-D-glucan and 2-amino-2-deoxy- $\beta$-D-glucan units. With its good cytocompatibility and interesting physicochemical properties, $\mathrm{CHI}$ has been applied in a lot of biomedical researches, 
including biomedical implant surface coating, tissue scaffold and nanomedicine. ${ }^{31-33}$ Various $\mathrm{CHI}$ nanoparticles were prepared using "polyelectrolyte complexation" (with anionic macromolecules) or "ionic gelation" (with anionic oligo-molecules) in the nanomedicine fields. ${ }^{31}$ In this study, drug-loaded $\mathrm{CHI}$ nanoparticles were prepared by the "ionic gelation" method, having advantages of the electrostatic complexation of the cationic $\mathrm{CHI}$ and anionic TPP, in which TPP is the most classic ionic agent in the CHI nanoparticle preparation. ${ }^{34}$ The antiproliferative drug DOX, which has been previously investigated for PCO prevention, was added in the $\mathrm{CHI}$ solution to obtain the drug-loaded CHI nanoparticles. ${ }^{35,36}$ As shown in Figure 2A, the N/P ratio was critical to control the nanoparticle size and its distribution in the uncross-linked case. In the investigated N/P ratio ranges (2-6), nanoparticles with diameter from $190 \mathrm{~nm}$ to $400 \mathrm{~nm}$ were obtained. It can be seen that by decreasing the N/P ratio, nanoparticles with smaller size were produced. It is reported that unstable large complex particle forms when N/P ratio around 1 , whereas the TPP condensation efficiency decreases in larger $\mathrm{N} / \mathrm{P}$ ratios, which may be due to the charge balance. ${ }^{22,35}$ The zeta potential investigation shows that the nanoparticles in these cases are strongly positively charged, ranging from $29.7 \mathrm{mV}$ in $\mathrm{N} / \mathrm{P}=2$ to $56.5 \mathrm{in} / \mathrm{P}=6$. The particle surface potential increases with increase in the N/P ratio, which may be attributed to the increasing of positively charged $\mathrm{CHI}$ on the nanoparticle. The $\mathrm{CHI}$ nanoparticle cross-linked via EDC/NHS chemistry was also investigated. After cross-linking, the particle size was more stable in investigated $\mathrm{N} / \mathrm{P}$ ratios, maintaining in $200 \mathrm{~nm}$ from $\mathrm{N} / \mathrm{P}=2$ to 5 . It is also shown that the zeta potentials slightly decrease after cross-linking. However, the obtained nanoparticles have a strong positive charge, ranging from $28.2 \mathrm{mV}$ to $40.5 \mathrm{mV}$. The chemical cross-linking will greatly improve the stability and the drug-sustained release properties of the nanoparticles. As a result, the N/P ratio of 2.5 and chemical cross-linking were selected in the subsequent investigations. The particle size and zeta potential are $203.4 \pm 2.5 \mathrm{~nm}$ and $33.4 \pm 0.9 \mathrm{mV}$, respectively. The particle size distributions of nanoparticles before or after chemical cross-linking are shown in Figure S1. They also indicated that the chemical cross-linking improved the particle size distribution. Unimodal distribution was found after chemical cross-linking. Such nanoparticles were also observed by SEM imaging. As shown in Figure 2B, nanoparticles with diameters around $200 \mathrm{~nm}$ are found, which confirms the success of nanoparticle preparation.

\section{Drug-Loaded Multilayer Fabrication And Coating Onto IOL}

HEP is also a naturally occurring linear polysaccharide, consisting primarily of two highly sulfated sugar monomers, which occur as repeating disaccharide units. As sharing chemical and structural similarity to heparan sulfate, a natural anticoagulant surface activity of the vascular endothelium, HEP also has excellent anticoagulant and

\section{A}
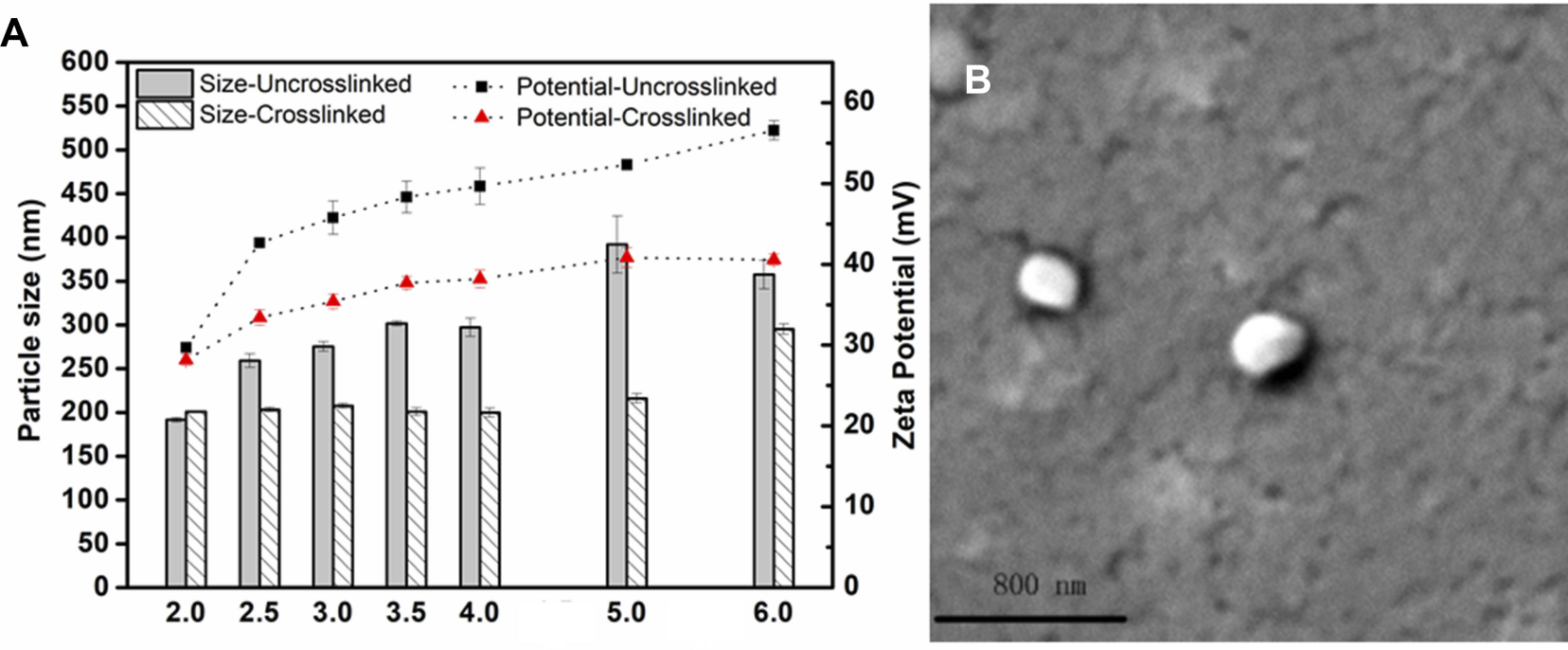

\section{N/P ratio}

Figure 2 (A) Chitosan particle sizes and zeta potentials change with N/P ratios. (B) Chitosan nanoparticle morphology observed by SEM. 
anti-adhesion properties. As a result, HEP is widely used in biomedical surface modification. ${ }^{7}$ In our previous research, $\mathrm{HEP} /$ collagen or $\mathrm{HEP} / \mathrm{CHI}$ multilayers have been constructed on the cardiovascular stent surface for anticoagulant and endothelialization. ${ }^{20,21,32,37,38}$ On the other hand, HEP is also used in IOL surface modifications. Till date, the heparinized IOL is the first and only approved clinically used surface-modified IOL. ${ }^{39,40}$ Taking advantages of its strong negative charge and distinctive properties in surface coating, HEP is used as one of the components for drug-eluting IOL surface modification. The freshly prepared cationic CHI-TPP-DOX nanoparticles (CTDNP) were LbL deposited with anionic HEP, obtaining drug-loaded polysaccharide multilayer on the IOL surface. The fabrication process of HEP/CTDNP multilayer was followed by QCM-D, which is a useful and powerful demonstration of the polyelectrolyte multilayer fabrication. ${ }^{13,20,21}$ QCM-D is a versatile and sensitive sensor technique to measure both adsorbed mass and the viscoelastic properties of adsorbed layers of molecules in liquid. ${ }^{13}$ The crystal sensor resonance frequency $(\mathrm{F})$ depends on the total oscillating mass, including coupled water to the oscillation. ${ }^{32}$ The $\mathrm{F}$ decreases when a thin film is attached to the crystal. ${ }^{21}$ Figure 3 shows $\mathrm{F}$ traces at different overtones $(\mathrm{v})$, where the $3 \mathrm{rd}, 5 \mathrm{th}, 7 \mathrm{th}, 9$ th and 11 th v corresponds to $15,25,35,45$ and $55 \mathrm{MHz}$, respectively. Step-by-step F shift is observed by injecting polyelectrolyte solution, which clearly illustrates a typical LbL buildup process of HEP/CTDNP multilayer. The ladder trace of frequency decrease indicates the successful adsorption of HEP or CTDNP onto the substrate alternatively. The (HEP/CTDNP $)_{5}$ multilayer was then introduced

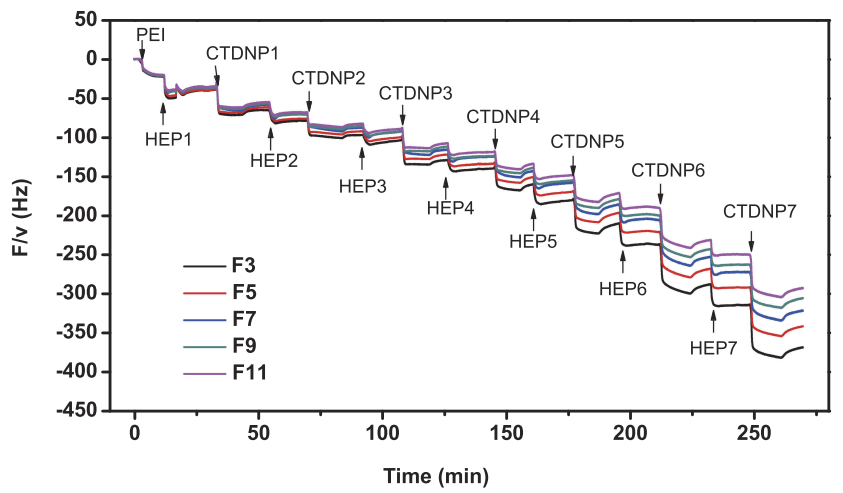

Figure 3 QCM-D shows the HEP/CTDNP multilayer deposition process on the surface. The arrows indicate the injection of different polyelectrolytes.

onto the IOL surface. As indicated in the stereomicroscopic image of the multilayered IOL (Figure 4B), LbL technique presents excellent feasibility to fabricate a homogenous thin coating onto the sophisticated 3-D implants. The commercialized hydrophobic IOL was used in present investigation. It is circular in shape with raised center, $6 \mathrm{~mm}$ in diameter of biconvex optic with two haptics. There are no obvious opaque arises after modification, compared with the unmodified IOLs (Figure 4A). The effects of the multilayer coating on the light transmission were then verified by UV-Vis spectra. As shown in Figure S2, the light transmittance of pristine IOL material was round $92 \%$. The surface modification of (HEP/ CTDNP $)_{5}$ multilayer just slightly decreases the light transmittance, with transmittance in the ranges of $85 \%$ to $92 \%$ for the visible light $(380 \mathrm{~nm}$ to $760 \mathrm{~nm})$. The (HEP/ CTDNP $)_{5}$ multilayer coating does not significantly influence its optical properties.
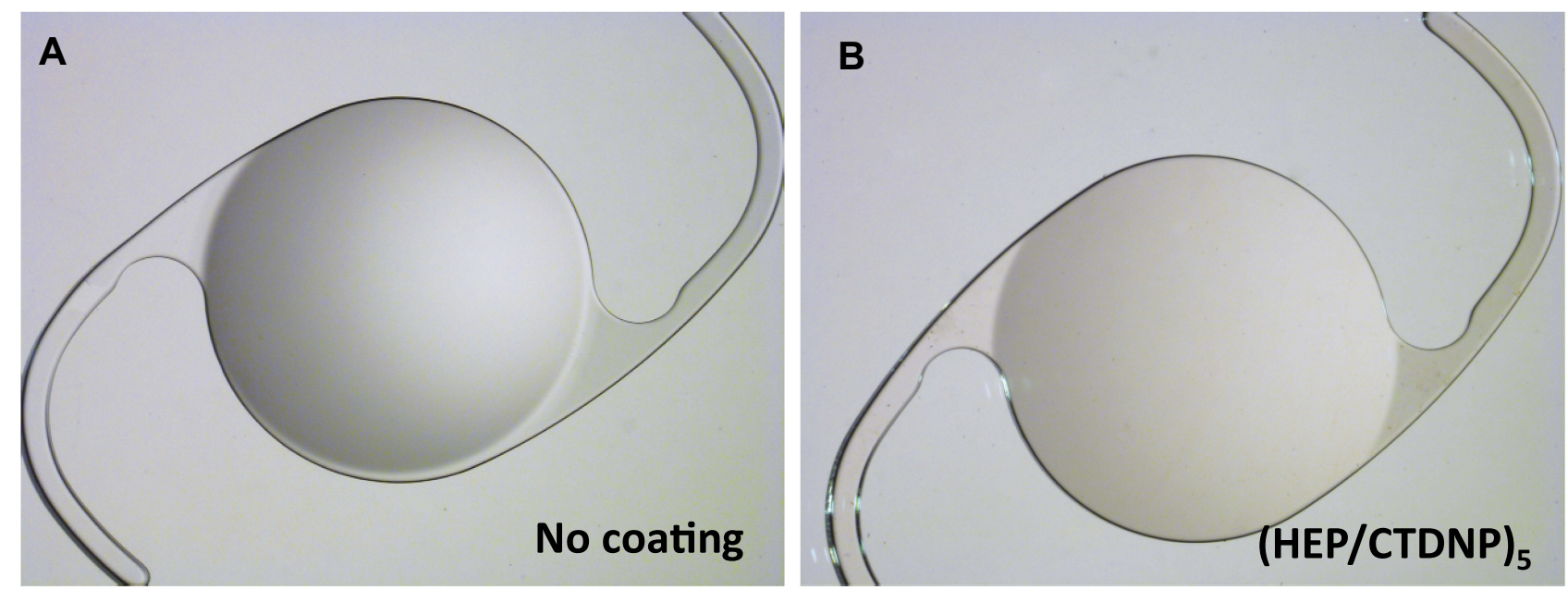

Figure 4 The stereomicroscopic images of the unmodified IOL (A) and the IOL after (HEP/CTDNP)5 coating modification (B). 


\section{Drug-Eluting Investigation}

DOX is one of the most frequently studied antiproliferative drugs in proliferative diseases, such as cancer nanomedicine and drug-eluting stents. ${ }^{41,42}$ Herein, DOX was selected as a model drug to prepare the drug-eluting IOLs. DOX was incorporated in the CHI-TPP nanoparticles and then LbL deposited onto the IOL surface. The drug release ability of the (HEP/CTDNP $)_{5}$ multilayer coating on the material surface was evaluated. As PCO was the result of the pathological hyperplasia of the residual LEC on the lens capsule and IOL surface, the release kinetics of the multilayer coating in both simulant physiological $(\mathrm{pH}=7.4)$ and pathological conditions $(\mathrm{pH}=5.5)$ was taken into consideration. $^{22}$ The surface-modified materials were soaked in the buffer at $37^{\circ} \mathrm{C}$ with gentle shaking. The releasing buffer was collected at appointed time points and analyzed by UPLC. As shown in Figure 5A, there is a burst release in the uncross-linked case under physiological condition $(\mathrm{pH}=7.4)$, whereas there is almost no drug release in the cross-linked one. There may be several reasons for this phenomenon. The most important one may be due to the unique charge and solubility changes of CHI in the physiological buffer. With a pKa around 6.5, $\mathrm{CHI}$ is protonated in an acidic environment. So it is positively charged and soluble when $\mathrm{pH}$ is lower than $\mathrm{pKa}$. When the $\mathrm{pH}$ increases under physiological conditions $(\mathrm{pH}=7.4)$, deprotonation takes place in the $\mathrm{CHI}$ chains, which results in its charge loss and insoluble. In uncross-linked cases, the charge loss of $\mathrm{CHI}$ in the nanoparticle may result in the breakdown of the polyelectrolyte multilayer, degelation of the ionic complexation and release of the drugs. However, in the cross-linked cases, the multilayer coating is stable when $\mathrm{pH}$ changes. What is more, the deprotonation of the CHI may further gel the multilayer coating, deprotonated $\mathrm{CHI}$ from physical crosslinking points in the coating, which may be the reason for the very small amount of drug release in the cross-linked case when $\mathrm{pH}$ is $5.5 .^{43}$ As mentioned earlier, the PCO development is mainly due to the adhesion and proliferation of the LEC. The cell proliferation under pathological conditions always results in the slightly acidic cellular microenvironment. ${ }^{44}$ Therefore, the drug release profile under pathological condition $(\mathrm{pH}=5.5)$ was evaluated. Sustained release profile was observed in this situation, as illustrated by Figure 5B. No burst release was found in both the cross-linked and uncross-linked multilayers. Moreover, the cross-linking further slows down the drug release. The accumulative drug release rate in the uncrosslinked one after 1 day is around $7.5 \%$ and it slowly increases to $11 \%$ after 1 week, whereas the release rates in the cross-linked multilayers are $4.4 \%$ and $7.2 \%$ after 1 day and 1 week, respectively. This result indicates that the drug-eluting IOL is stable under physiological condition and renders sustained drug release property under pathological condition.

\section{LECs Adhesion, Proliferation And \\ Migration}

The LECs adhesion, proliferation and migration are the key points in the PCO development. Herein, the in vitro
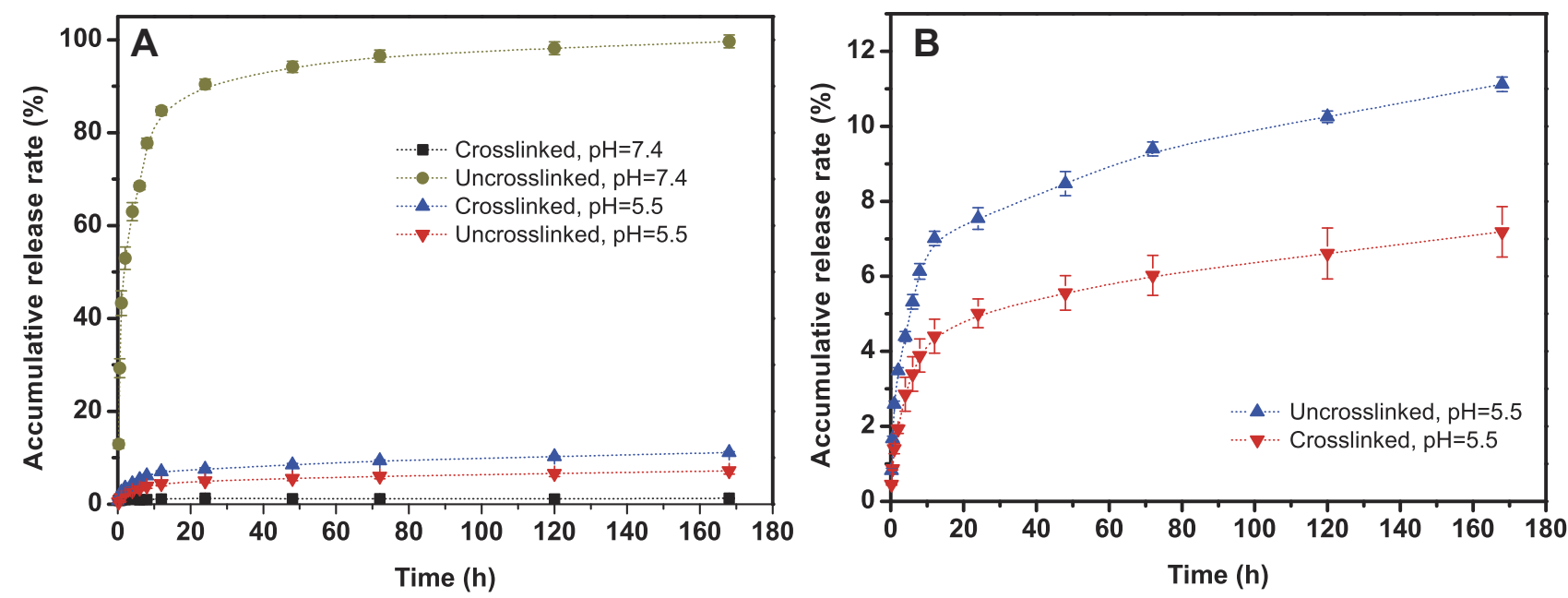

Figure 5 (A) Accumulative drug releases from the drug-eluting $\mathrm{IOL}$ with and without cross-linking under different $\mathrm{pH}$ conditions. Black square and indigo circle refer to the drug releasing from the cross-linked and uncross-linked coatings under $\mathrm{pH} 7.4$, respectively. Blue triangle and red invert triangle refer to the drug releasing from the crosslinked and uncross-linked coatings under $\mathrm{pH}$ 5.5, respectively. (B) Amplified image of the releasing profiles under $\mathrm{pH} 5.5$. 
cell behaviors of the drug-eluting IOLs were tested. Figure 6 shows the quantitative results of the cell adhesion and cell apoptosis on the different surfaces compared with tissue culture plates (TCP) by live and death stain method. Cell adhesion is greatly inhibited after surface coating with antiproliferative drug-loaded polysaccharides multilayer, as illustrated in Figure 6A. On the unmodified IOL materials, the adhesion rate is comparable to the TCP $(95.6 \pm 15.6 \%)$. Surface modification with HEP ( 0.5 bilayer) decreases the cell adhesion, with adhesion rate around $56.7 \% \pm 9.8 \%$. The drug-loaded nanoparticle deposition or the subsequent (HEP/ CTDNP) bilayer cycle modification further inhibits the surface cell adhesion, with cell adhesion rates lower than $10 \%$. There was a statistically significant difference in cell adhesion rate between the unmodified IOL and the IOL modified with different layers. The cell adhesion rate of the HEP modification ((HEP/CTDNP $\left.)_{0.5}\right)$ IOL was also statistically different from that of the 1 layer, 4.5 layer and 5 layers, indicating that the modified IOL can significantly reduce the adhesion of LECs on the IOL surface. Figure 6B indicates the apoptosis rates of the adherent cells on the surfaces. The apoptotic rate of LECs on unmodified IOL surface had no statistical difference from the only HEP modification ((HEP/ CTDNP) $)_{0.5}$ ) or the lower deposition cycle modification (e.g. (HEP/CTDNP $)_{1}$ ), indicating that the simple antiadhesion modification of HEP sodium and insufficient drug loading could not significantly induce the apoptosis of LECs. However, the LECs apoptotic rate of the $(\mathrm{HEP} / \mathrm{CTDNP})_{4.5}$ and $(\mathrm{HEP} / \mathrm{CTDNP})_{5}$ multilayer- modified IOL was statistically different from the unmodified IOL and HEP-modified IOL. When (HEP/ CTDNP) deposition cycle increases, the apoptotic cell proportion increases in the adherent cells, with apoptosis rates of $30-50 \%$. Figure $7 \mathrm{~A}$ shows the representative images of the living cells on these surfaces, which directly renders the adherent cell distributions and morphologies on the unmodified or different bilayers of (HEP/CTDNP) multilayer modified IOL material surfaces. The cell spreads well on the unmodified surface or the coatings with HEP as the outmost layer. However, despite the decreased adherent cell number on the drugloaded nanoparticle as the outmost layers, the rare adherent cells present un-spreading morphologies, which also indicates the adherent cells are apoptotic on this surface. In our previous researches, the hydrated polysaccharide multilayer coating can reduce the nonspecific cell adhesion on the material surface, yet it does not decrease the PCO incidence in the long run. ${ }^{13}$ There may be several reasons due to the complex environment in vivo. Although the polysaccharide multilayer coating effectively resists the LEC initial adhesion on the IOL surface, cells can still adhere onto the capsular membrane, as the lens capsular bag is a relatively closed space. Then, the subsequent cell proliferation and migration between capsular membrane and IOL may happen, which results in the PCO. Thus, such anti-adhesion modification cannot eliminate PCO in vivo, but only delay its developing time. As a result, the antiproliferative drug loading to inhibit cell proliferation in the long run is essential. In the present investigation, there are

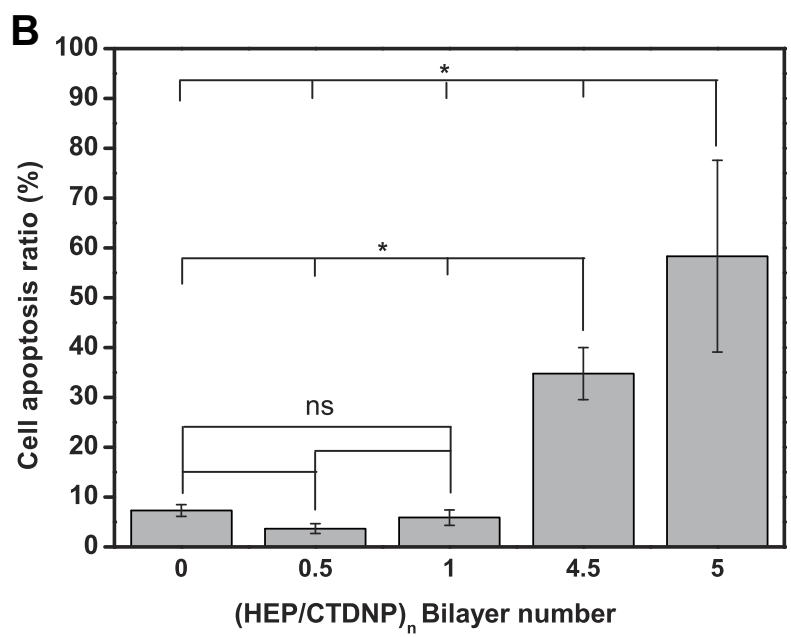

Figure 6 (A) Cell adhesion ratios on the material surface with different bilayer multilayer modifications. (B) Cell apoptosis ratios on the material surface with different bilayer multilayer modifications. ${ }^{*} P<0.05$, ns means no statistical significance. 


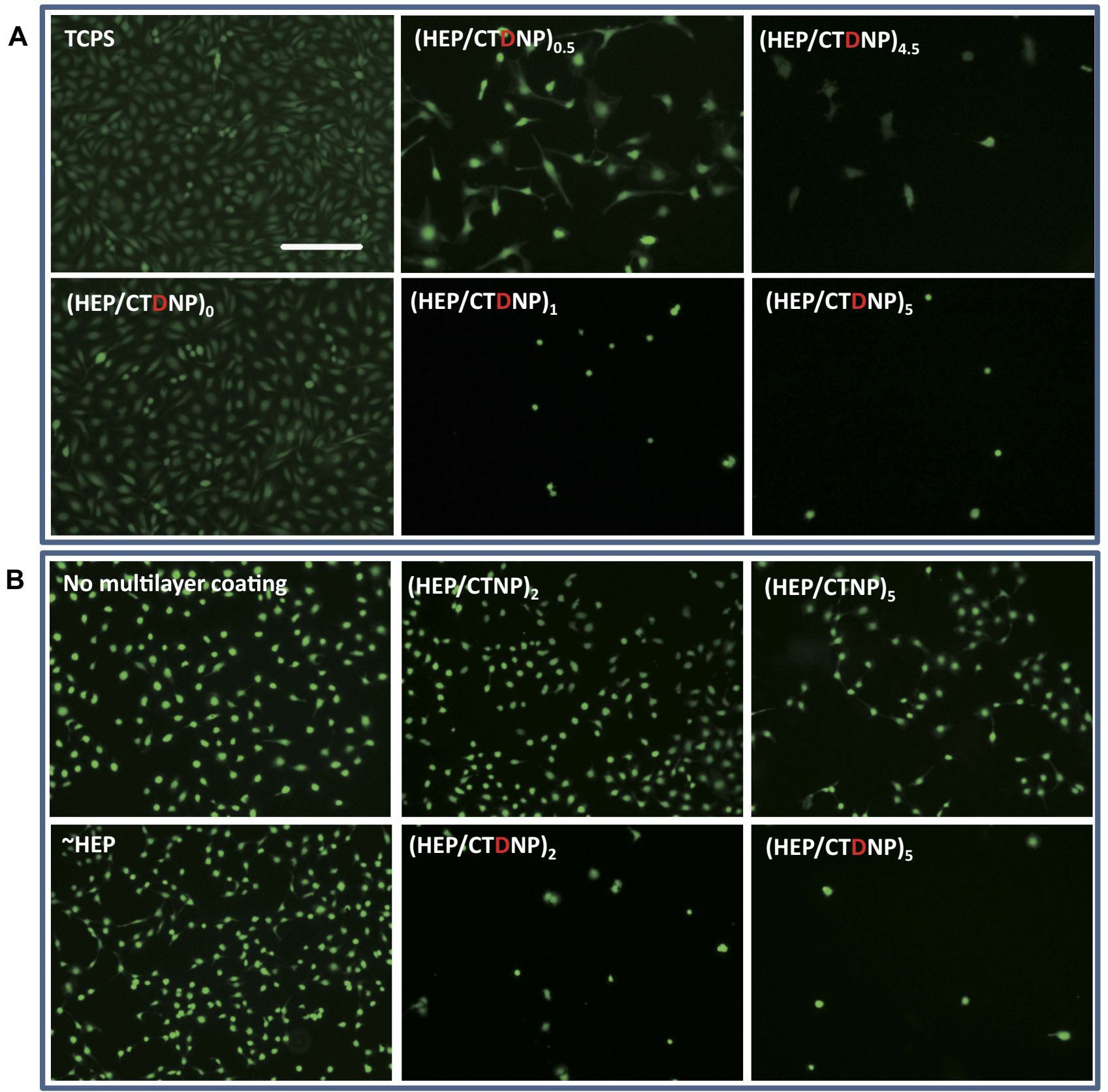

Figure 7 (A) Cell distributions and morphologies on the material surface with different bilayer multilayer modifications. (B) Cell distributions on the material surface with different bilayer multilayer modifications with drug loading $\left((\mathrm{HEP} / \mathrm{CTDNP})_{\mathrm{n}}\right)$ or without drug loading $\left((\mathrm{HEP} / \mathrm{CTNP})_{\mathrm{n}}\right)$. Scale bar=200 $\mu \mathrm{m}$.

very few cells adherent on the material surface even after the one bilayer coating, which may be combined effects of the anti-adhesion of the polysaccharide multilayer and the antiproliferative drug in the coatings. To confirm this hypothesis, the CHI nanoparticles without DOX were prepared and the multilayer coatings without drug loading were fabricated. The cell adhesions on these surfaces were compared. As shown in Figure 7B, the cell adhesion is decreased with the multilayer increasing, whereas there are considerable number of adherent cells on the HEP/CTNP surface, even after five bilayer coatings. As the hyaluronic acid (HA) is the most hydrated polysaccharide among the glycosaminoglycan, it can be seen that the cell resistant property of the HEP/CHI nanoparticle multilayer is not as well as the $\mathrm{HA} / \mathrm{CHI}$ multilayer in our previous investigations, where the cell adhesion is negligible after five bilayer modifications. ${ }^{13}$ The antiproliferative drug loading evidently decreases the adherent cells on the HEP/CTDNP multilayer coatings, even after the first one or two 
deposition cycles; this results in the induction of cell apoptosis by the antiproliferative drugs in these surface coatings.

To evaluate the effect of the surface modification on cell inhibition in the capsular bags, the cell migration experiment was carried out. Cells were seeded on the peripheral parts of the cell culture wells and the materials with or without drug-loaded multilayer coating were placed in the central areas. The effect of the drug-loaded multilayer on the LEC migration was observed in the photograph. As shown in Figure 8, the cells migrated into the central part of the culture wells when no material was placed (Figure 8B). When the IOL material was placed, cell migration was suppressed, but not inhibited. The cells grow well on the peripheral area and few cells migrate into the central area (Figure $8 \mathrm{C}$ ). When the drug-eluting IOL material was
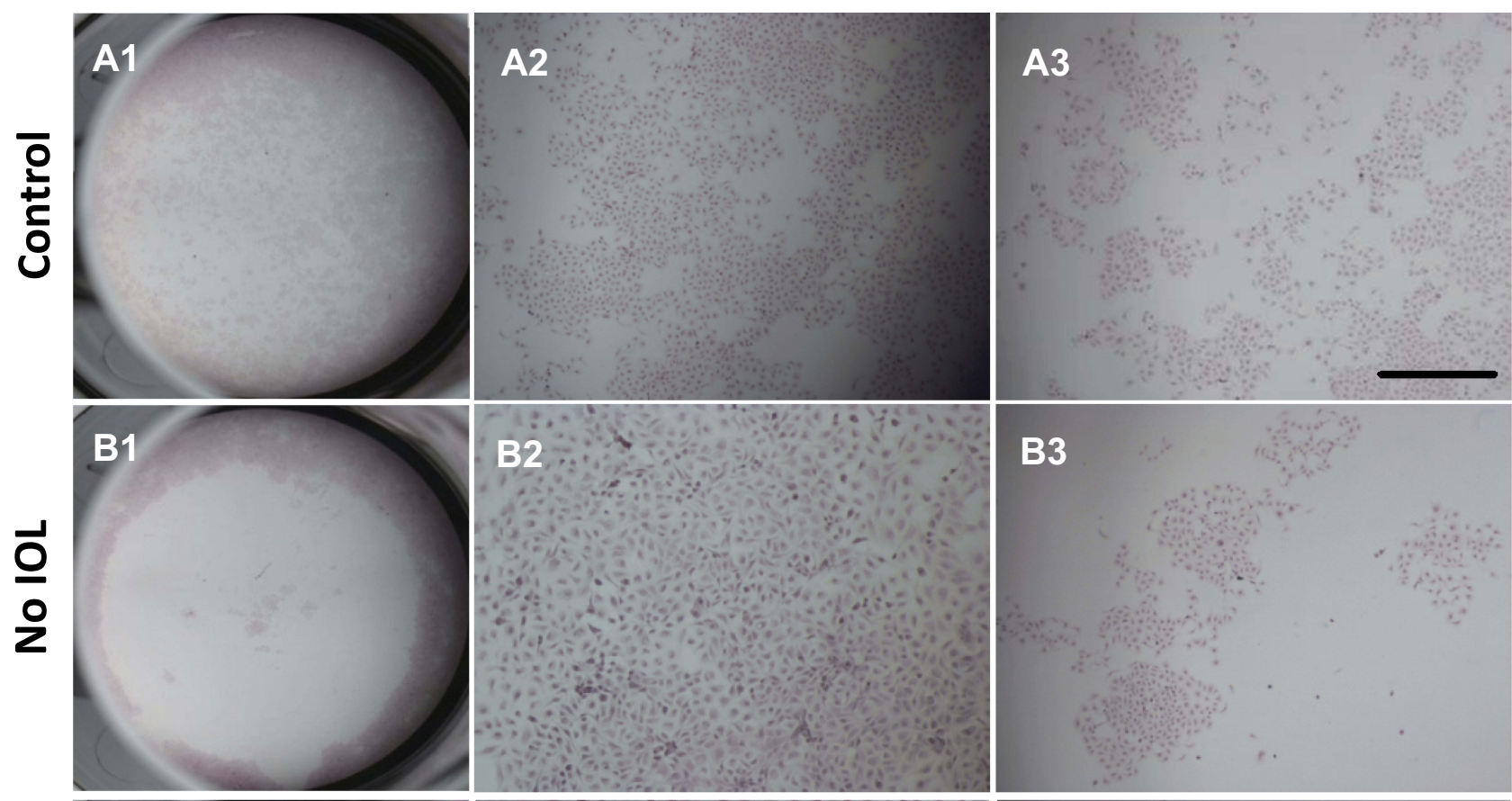

\section{B3}
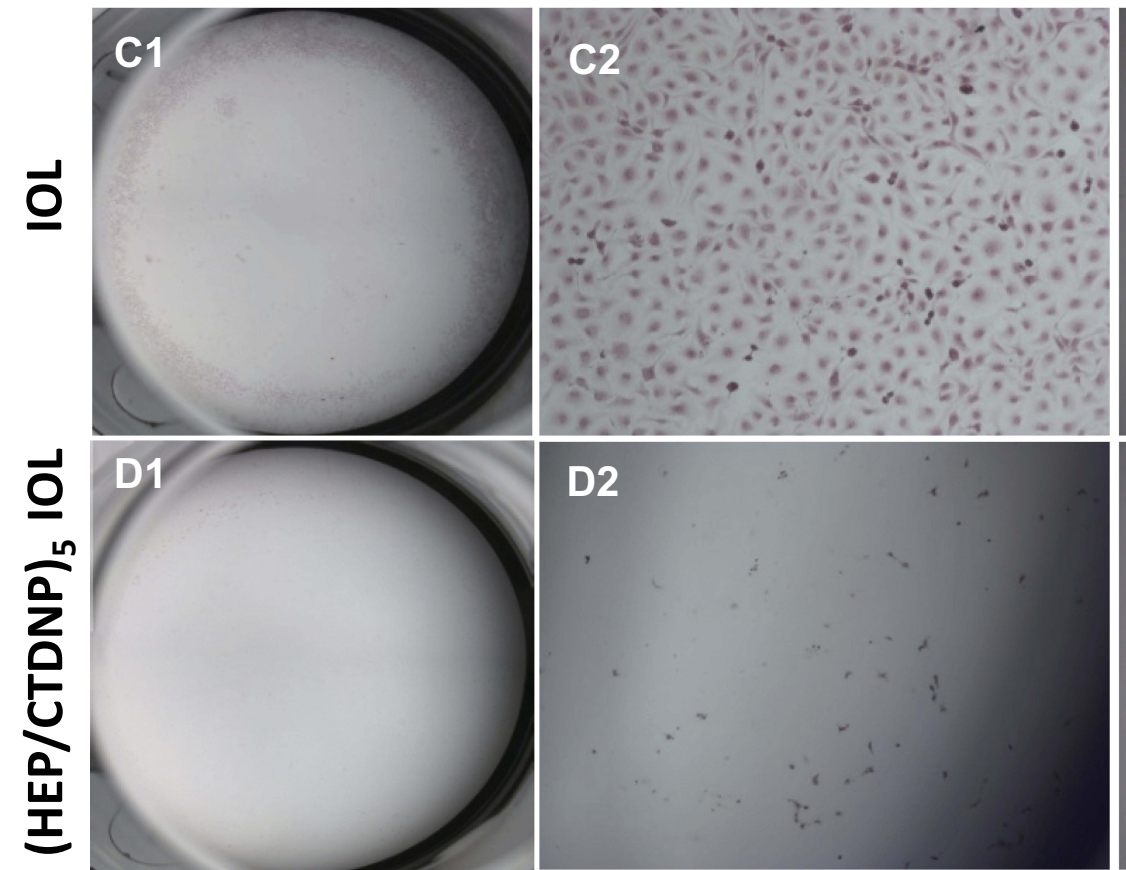

\section{D2}

\section{C3}
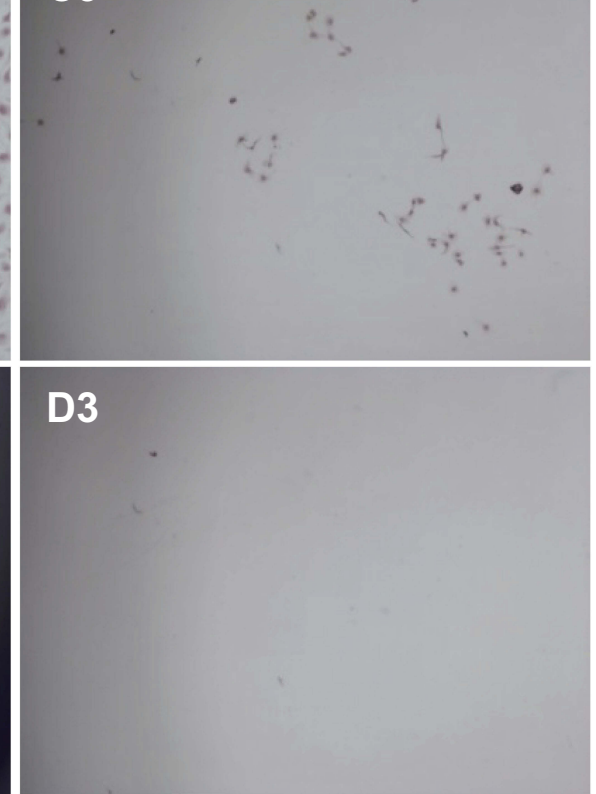

Figure 8 Cell migration assays via cells seeding under different conditions. Images AI-DI are the stitched images of the 24-well cell culture plates. Images A2-D2 are the representative photos taken from the peripheral area and images A3- D3 are the representative photos taken from the central area. Scale bar=200 $\mu \mathrm{m}$. 
placed, not only the cell migration is inhibited but also the peripheral cell apoptosis was obtained under this circumstance (Figure 8D). The IOL with drug-loaded multilayer coatings presents excellent cell adhesion inhibition, apoptosis inducing and the cell migration suppressing properties, which may have great potential for PCO inhibition in vivo.

\section{In Vivo Anti-PCO Evaluation}

The in vivo PCO inhibition effects of the drug-eluting IOLs were evaluated by the intraocular implantation after phacoemulsification surgery was performed in rabbit eyes. The slit lamp examinations were performed at 1 day, 1 week, 2 weeks and 2 months postoperatively. The anterior chamber inflammations in both groups were alleviated in 1 day after the surgery (Figure 9A1, B1). There are no cell adhesion in both groups postoperative 1 week (Figure 9A2, B2). Posterior capsule starts
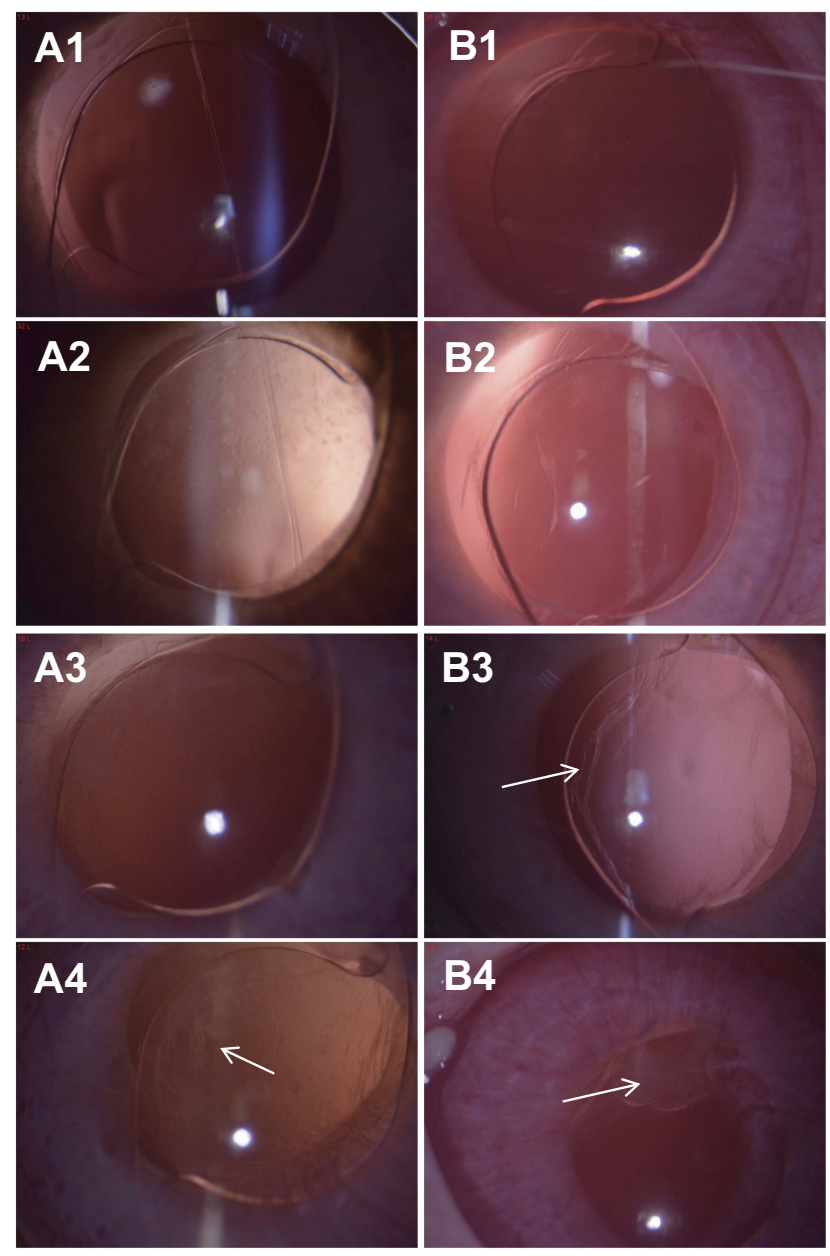

Figure 9 Slit lamp micrographs of IOL with (A) or without (B) (HEP/CTDNP) multilayer modification in rabbit eye. Images $A I / B I$ to $A 4 / B 4$ are obtained at postoperative I day, I week, 2 weeks and 2 months postoperatively. The white arrows indicate the PCO. shrinking in control eyes at 2 weeks postoperatively, showing localized capsular folds and fibrosis (white arrow in Figure 9B3). The PCO develops rapidly after its appearance. At 2 months postoperatively, most control eyes show obvious epithelial opaque and pearl-type PCO (white arrow in Figure 9B4). The posterior synechia of the iris is also found in the control groups, as the pupil can not be dilated completely after dripping with mydriasis (Figure 9B4). By contrast, no capsular shrinking is observed in the drug-eluting IOL group at 2 weeks after surgery (Figure 9A3). No PCO develops in the drug-eluting IOL group at postoperative 2 months except one eye shows mild capsular shrinking (Figure 9A4). The effect of the surface coating on PCO inhibition was further evaluated by the gross examination and PCO scoring. The animals were sacrificed humanely after euthanasia after 10 weeks. The eye was then sectioned at the equator. Gross examination of the anterior segment was performed from the posterior aspect (Miyake-Apple View) to assess the degree of the capsular opacification. The textures of the peripheral iris, where the capsular bags are not covered, are clearly visible in both control group (Figure 10B1) and experimental group (Figure 10A1, red dash circle indicates the IOL edges of the main parts). Iris and pupil can be clearly seen through the capsule in the drug-eluting IOL implanted cases (Figure 10A1), whereas serious opacification is found in the capsular bag in the unmodified IOL groups (Figure 10B1). Serious fibrosis is observed in the unmodified IOL samples. The
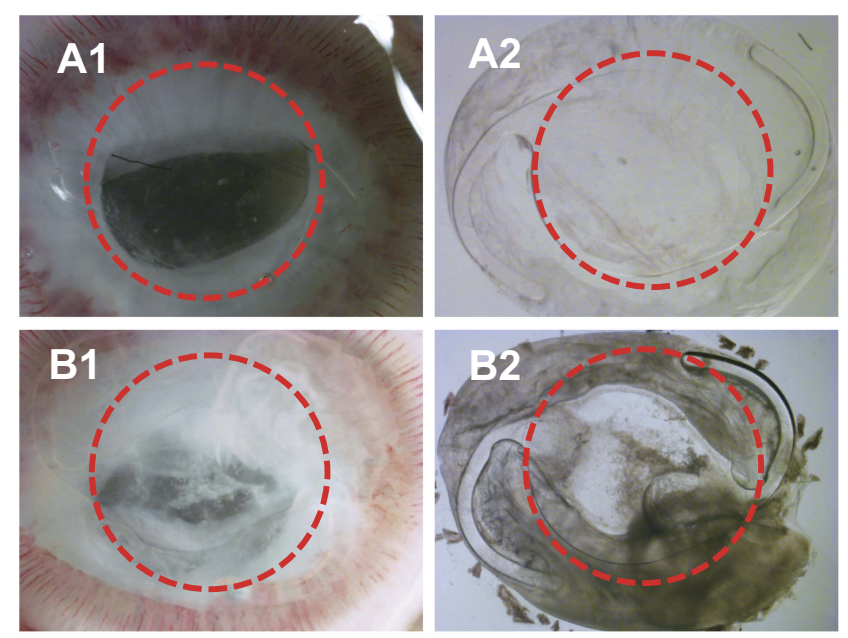

Figure 10 Gross photographs from the posterior (Miyake-Apple view) of rabbit eyes implanted with drug-eluting $I O L(A I)$ or pristine $I O L(B I)$. The anterior view of the separated lens capsules with drug-eluting IOL (A2) or pristine IOL (B2). The red dotted circles indicate the circular outer periphery of the IOL. 
stereomicroscopic images clearly show the SR and the posterior capsular opaque phenomena (Figure 10A2, B2). In the drug-eluting coating-modified IOL cases, the capsular bag with IOL is clear. Most of the optic and haptic parts of IOL keep transparent and only mild SR developed in the bag edges (Figure 10A2). However, in the pristine IOL cases, the posterior capsule opacification on IOL starts at the optic-haptic junctions and invades into the IOL optic center. Serious SR formation and posterior capsular turbidity are found in the capsular bags (Figure 10B2). The histological sections of the different lens capsules after 10 weeks of IOL implantation directly illustrate the PCO developments. The heavy $\mathrm{SR}$, as well as serious anterior and posterior capsular hyperplasia, is found in the unmodified IOL cases (Figure 11B1, B2). However, the mild SR formation and very slight anterior and posterior capsular hyperplasia occur in the drug-eluting coating modification IOL cases (Figure 11A1, A2). Figure 12 shows the quantitative SR and PCO scores of both groups. In the animal PCO models, the unmodified IOLs show high PCO incidence. The scores of SR, PPCO and CPCO are $2.29 \pm 028,1.90 \pm 0.68$ and $1.11 \pm 0.79$, respectively. The drug-eluting coating modification decreases the PCO scores, in which SR, PPCO and CPCO scores are 0.73 $\pm 0.27,0.44 \pm 0.34$ and $0.33 \pm 0.31$, respectively. The drugeluting coating modification significantly decreases the PCO incidence.

As shown in the 'Results and Discussion' section, in unmodified cases, heavy proliferation of the residual LECs

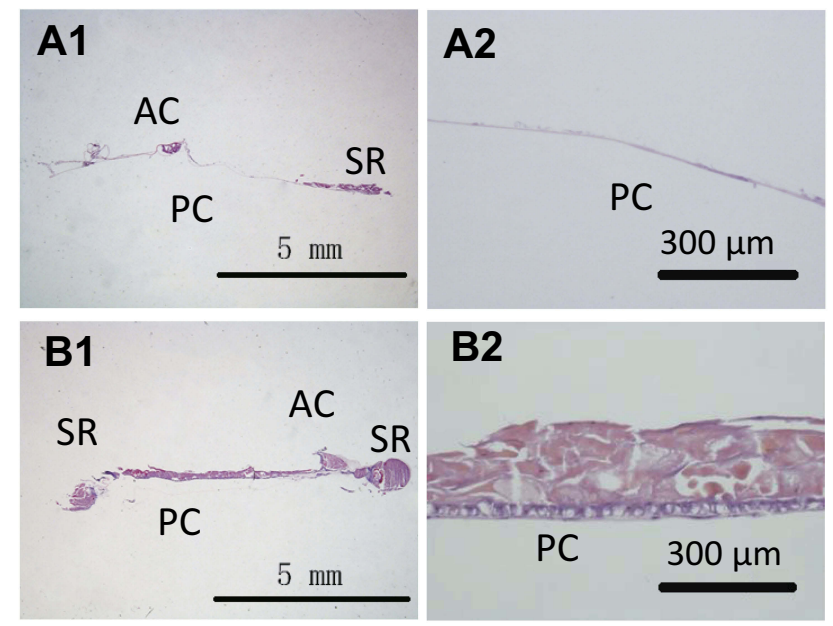

Figure II Photographs of histological sections cut from lens capsular bag with drug-eluting $I O L(A I)$ and pristine $I O L(B I)$ implantation. $A 2$ and $B 2$ images are the amplification of the $\mathrm{AI}$ and $\mathrm{B} 2$ of the posterior capsule.

Abbreviations: SR, Soemmering's ring; AC, anterior capsule; PC, posterior capsule.

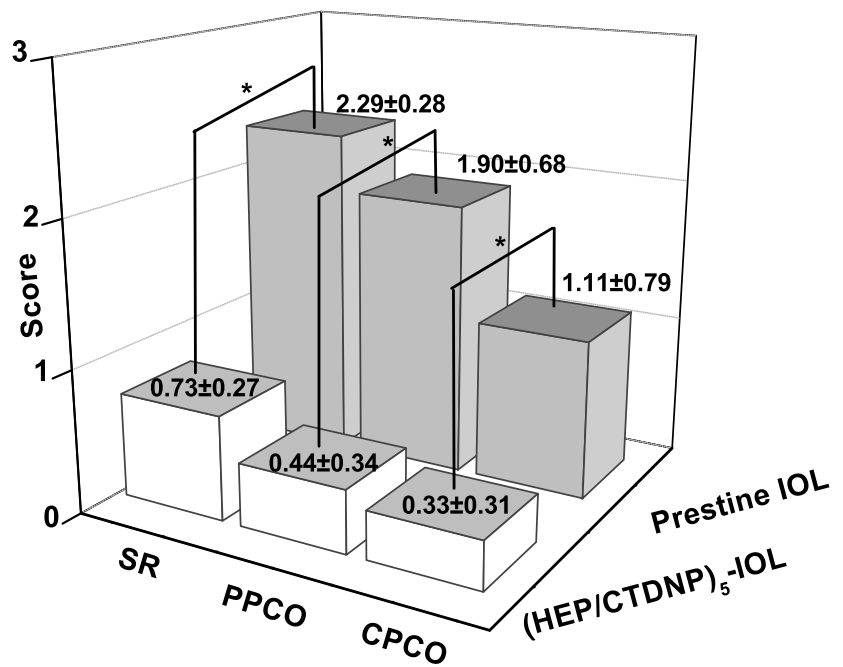

Figure 12 Soemmering's ring (SR), peripheral PCO (PPCO) and central PCO $(\mathrm{CPCO})$ scores of drug-eluting IOL $\left((\mathrm{HEP} / \mathrm{CTDNP})_{5}\right.$ multilayer modified) and control pristine IOL $(* P<0.05)$.

takes place and SR started to proliferate onto the posterior capsules and thick hyperplasia is observed between posterior capsule and IOL surface. In contrast, the cell migration and proliferation were greatly inhibited in the drug-eluting hydrophilic coating-modified IOL cases. Very tiny SR formation was found and almost no posterior capsule hyperplasia was developed under this condition. Compared with unmodified IOLs or hydrophilic anti-adhesive coating-modified IOLs in previous investigations, the current drug-eluting hydrophilic coating modification effectively decreases the PCO incidence after implantation. ${ }^{13-16,22}$ The drug-eluting coating may have a great impact on the prevention of IOL postoperative complication after cataract surgery.

\section{Conclusion}

In conclusion, antiproliferative drug-loaded nanoparticle incorporated polysaccharide multilayer was constructed and fabricated onto the IOL surface for IOL postoperative PCO incidence prevention. The DOX-loaded CHI nanoparticles with strongly positive charges were successfully prepared via ionic gelation method on the particle surface. The drug-eluting coatings were obtained from the LbL electrostatic deposition of the positively charged nanoparticle and the negatively charged HEP. In vitro cell adhesion was reduced. The cell migration and proliferation were remarkably inhibited due to the hydrophilic hydration and the antiproliferative drug-eluting properties, respectively. Combined the cell resist coating with antiproliferative properties, such drug-eluting hydrophilic polysaccharide multilayer coating surface-modified IOLs 
evidently inhibit the postoperative PCO development. The in vivo rabbit model demonstrates that the antiproliferative drug nanoparticle-incorporated hydrophilic polysaccharide multilayer-modified IOLs (drug-eluting IOLs) significantly inhibit PCO formation after IOL implantation, either in SR formation, PPCO or CPCO. Taking advantages of the antiadhesion and anti-proliferation, such multifunctional surface-modified IOLs have higher in vivo biocompatibility compared with hydrophilic surface modified or pristine ones. However, the accelerated rabbit PCO model used for in vivo studies may not be predictive of human response. Therefore, human clinical investigation may further be carried out to demonstrate the benefits of this technology.

\section{Acknowledgments}

This work was supported by National Key R\&D Program (2017YFC1104602), National Natural Science Foundation of China (31570959, 81771984), Medical \& Health Technology Program of Zhejiang Province (2018KY540), Basic Scientific Program of Wenzhou (Y20180170) and the Top-notch Youth Talent of "Ten Thousand Talents Program" of Zhejiang Province.

\section{Disclosure}

The authors declare no conflicts of interest in this work.

\section{References}

1. Bourne RRA, Flaxman SR, Braithwaite T, et al. Magnitude, temporal trends, and projections of the global prevalence of blindness and distance and near vision impairment: a systematic review and metaanalysis. Lancet Global Health. 2017;5(9):e888-e897. doi:10.1016/ S2214-109X(17)30293-0

2. Schmidbauer JM, Vargas LG, Peng Q, et al. Posterior capsule opacification. Int Ophthalmol Clin. 2001;41(3):109-131. doi:10.1097/ 00004397-200107000-00010

3. Marcantonio JM, Vrensen GFJM. Cell biology of posterior capsular opacification. Eye. 1999;13:484-488. doi:10.1038/eye.1999.126

4. Garty S, Shirakawa R, Warsen A, et al. Sustained antibiotic release from an intraocular lens-hydrogel assembly for cataract surgery. Invest Ophthalmol Vis Sci. 2011;52:6109-6116. doi:10.1167/iovs.10-6071

5. Lipnitzki I, Bronshtein R, Ben Eliahu S, Marcovich AL, Kleinmann G. Hydrophilic acrylic intraocular lens as a drug delivery system: influence of the presoaking time and comparison to intracameral injection. J Ocul Pharmacol Th. 2013;29:414-418. doi:10.1089/jop.2012.0062

6. Viola GM, Rosenblatt J, Raad II. Drug eluting antimicrobial vascular catheters: progress and promise. Adv Drug Deliver Rev. 2017;112:3547. doi:10.1016/j.addr.2016.07.011

7. Biran R, Pond D. Heparin coatings for improving blood compatibility of medical devices. Adv Drug Deliver Rev. 2017;112:12-23. doi:10.1016/j.addr.2016.12.002

8. Eloy R, Parrat D, Duc TM, Legeay G, Bechetoille A. In vitro evaluation of inflammatory cell response after CF4 plasma surface modification of poly(methyl methacrylate) intraocular lenses. J Cataract Refr Surg. 1993;19:364-370. doi:10.1016/S0886-3350(13)80307-0
9. Yammine P, Pavon-Djavid G, Helary G, Migonney V. Surface modification of silicone intraocular implants to inhibit cell proliferation. Biomacromolecules. 2005;6:2630-2637. doi:10.1021/bm05 80101

10. Huang XD, Yao K, Zhang Z, Zhang Y, Wang Y. Uveal and capsular biocompatibility of an intraocular lens with a hydrophilic anterior surface and a hydrophobic posterior surface. J Cataract Refr Surg. 2010;36:290-298. doi:10.1016/j.jcrs.2009.09.027

11. Huang X, Luo C, Lin L, et al. UV-assisted treatment on hydrophobic acrylic IOLs anterior surface with methacryloyloxyethyl phosphorylcholine: reducing inflammation and maintaining low posterior capsular opacification properties. Mat Sci Eng C-Mater. 2017;75:12891298. doi:10.1016/j.msec.2017.03.029

12. Krall EM, Arlt EM, Jell G, Strohmaier C, Moussa S, Dexl AK. Prospective randomized intraindividual comparison of posterior capsule opacification after implantation of an IOL with and without heparin surface modification. J Refract Surg. 2015;31:466-472. doi:10.3928/1081597X-20150623-05

13. Lin QK, Xu X, Wang BL, et al. Hydrated polysaccharide multilayer as an intraocular lens surface coating for biocompatibility improvements. J Mater Chem B. 2015;3:3695-3703. doi:10.1039/C5TB00 $111 \mathrm{~K}$

14. Xu X, Tang JM, Han YM, Wang W, Chen H, Lin QK. Surface PEGylation of intraocular lens for PCO prevention: an in vivo evaluation. J Biomater Appl. 2016;31:68-76. doi:10.1177/08853282166 38547

15. Han YM, Xu X, Tang JM, Shen CH, Lin QK, Chen H. Bottom-up fabrication of zwitterionic polymer brush on intraocular lens for improved biocompatibility. Int J Nanomed. 2017;12:127-135. doi:10. 2147/IJN.S107491

16. Lin QK, Tang JM, Han YM, Xu X, Hao XJ, Chen H. Hydrophilic modification of intraocular lens via surface initiated reversible addition-fragmentation chain transfer polymerization for reduced posterior capsular opacification. Colloid Surface B. 2017;151:271-279. doi:10.1016/j.colsurfb.2016.12.028

17. Lin QK, Xu X, Wang Y, Wang B, Chen H. Antiadhesive and antibacterial polysaccharide multilayer as IOL coating for prevention of postoperative infectious endophthalmitis. Int $J$ Polym Mater. 2017;66:97-104. doi:10.1080/00914037.2016.1190925

18. Tsai M, Bai S, Chen R. Cavitation effects versus stretch effects resulted in different size and polydispersity of ionotropic gelation chitosan-sodium tripolyphosphate nanoparticle. Carbohydr Polym. 2008;71:448-457. doi:10.1016/j.carbpol.2007.06.015

19. Lin QK, Ren KF, Ji J. Hyaluronic acid and chitosan-DNA complex multilayered thin film as surface-mediated nonviral gene delivery system. Colloid Surface B. 2009;74:298-303. doi:10.1016/j. colsurfb.2009.07.036

20. Lin QK, Van JJ, Qiu FY, Song XX, Fu GS, Ji JA. Heparin/collagen multilayer as a thromboresistant and endothelial favorable coating for intravascular stent. J Biomed Mater Res A. 2011;96A:132-141. doi: $10.1002 / \mathrm{jbm} . \mathrm{a} .32820$

21. Lin QK, Ding X, Qiu FY, Song XX, Fu GS, Ji J. In situ endothelialization of intravascular stents coated with an anti-CD34 antibody functionalized heparin-collagen multilayer. Biomaterials. 2010; 31:4017-4025. doi:10.1016/j.biomaterials.2010.01.092

22. Han Y, Xu X, Wang Y, et al. Drug eluting intraocular lens surface modification for PCO prevention. Colloid Interf Sci Commun. 2018;24:40-44. doi:10.1016/j.colcom.2018.03.007

23. Vieira AP, Pimenta AF, Silva D, et al. Surface modification of an intraocular lens material by plasma-assisted grafting with 2-hydroxyethyl methacrylate (HEMA), for controlled release of moxifloxacin. Eur J Pharm Biopharm. 2017;120:52-62. doi:10.1016/j. ejpb.2017.08.006

24. Huang Q, Cheng GPM, Chiu K, Wang GQ. Surface modification of intraocular lenses. Chinese Med J-Peking. 2016;129:206-214. doi:10.4103/0366-6999.173496 
25. Chehimi MM, Lamouri A, Picot M, Pinson J. Surface modification of polymers by reduction of diazonium salts: polymethylmethacrylate as an example. J Mater Chem C. 2014;2:356-363. doi:10.1039/C3T C31492H

26. Amoozgar B, Fitzpatrick SD, Sheardown H. Effect of anti-TGF- 2 surface modification of polydimethylsiloxane on lens epithelial cell markers of posterior capsule opacification. J Bioact Compat Pol. 2013;28:637-651. doi:10.1177/0883911513504855

27. Hettlich HJ, Otterbach F, Mittermayer C, Kaufmann R, Klee D. Plasma-induced surface modifications on silicone intraocular lenses: chemical analysis and in vitro characterization. Biomaterials. 1991;12:521-524. doi:10.1016/0142-9612(91)90153-2

28. Tang JM, Han YM, Chen H, Lin QK. Bottom-up fabrication of PEG brush on poly(dimethylsiloxane) for antifouling surface construction. Int J Polym Sci. 2016;2016:8458752. doi:10.1155/2016/8458752

29. Saika S, Werner L, Lovicu FJ. Lens Epithelium and Posterior Capsular Opacification. Japan: Springer; 2014.

30. Daemen J, Wenaweser P, Tsuchida K, et al. Early and late coronary stent thrombosis of sirolimus-eluting and paclitaxel-eluting stents in routine clinical practice: data from a large two-institutional cohort study. Lancet. 2007;369:667-678. doi:10.1016/S0140-6736(07)603 14-6

31. Mohammadpourdounighi N, Behfar A, Ezabadi A, Zolfagharian H, Heydari M. Preparation of chitosan nanoparticles containing Naja naja oxiana snake venom. Nanomed-Nanotechnol. 2010;6:137-143. doi:10.1016/j.nano.2009.06.002

32. Lin QK, Hou Y, Ren KF, Ji J. Selective endothelial cells adhesion to Arg-Glu-Asp-Val peptide functionalized polysaccharide multilayer. Thin Solid Films. 2012;520:4971-4978. doi:10.1016/j.tsf.2012.03.041

33. Masuko T, Iwasaki N, Yamane S, et al. Chitosan-RGDSGGC conjugate as a scaffold material for musculoskeletal tissue engineering. Biomaterials. 2005;26:5339-5347. doi:10.1016/j.biomaterials.2005.01. 062

34. Bugnicourt L, Ladavière C. Interests of chitosan nanoparticles ionically cross-linked with tripolyphosphate for biomedical applications. Prog Polym Sci. 2016;60:1-17. doi:10.1016/j.progpolymsci.2016. 06.002
35. Janes KA, Fresneau MP, Marazuela A, Fabra A, Alonso MJ. Chitosan nanoparticles as delivery systems for doxorubicin. J Control Release. 2001;73:255-267. doi:10.1016/S0168-3659(01)00294-2

36. Guha R, Chowdhury S, Palui H, et al. Doxorubicin-loaded MePEGPCL nanoparticles for prevention of posterior capsular opacification. Nanomedicine-UK. 2013;8:1415-1428. doi:10.2217/nnm.12.175

37. Lin QK, Yan JJ, Ji J, Shen JC. Natural anticoagulant and endothelial cell-compatible multilayer for endovascular stents surface modification. Chem J Chinese U. 2009;30:1256-1258.

38. Lin QK, Hou Y, Xu X, et al. Anti-CD34 antibody functionalized swollen polymeric coating for endothelial cell rapid selectively capture. Int J Polym Mater. 2015;64:99-103. doi:10.1080/00914037. 2014.886248

39. Larsson R, Selén G, Björdklund H, Fagerholm P. Intraocular PMMA lenses modified with surface-immobilized heparin: evaluation of biocompatibility in vitro and in vivo. Biomaterials. 1989;10:511-516. doi:10.1016/0142-9612(89)90055-0

40. Trocme SD, Li H-I. Effect of heparin-surface-modified intraocular lenses on postoperative inflammation after phacoemulsification: a randomized trial in a United States patient population. Ophthalmology. 2000;107:1031-1037. doi:10.1016/S0161-6420(00) 00098-1

41. Shi J, Kantoff PW, Wooster R, Farokhzad OC. Cancer nanomedicine: progress, challenges and opportunities. Nat Rev Cancer. 2017;17:2037. doi:10.1038/nrc.2016.108

42. Tsuruta W, Tsurushima H, Yamamoto T, Suzuki K, Yamazaki N, Matsumura A. Application of liposomes incorporating doxorubicin with sialyl Lewis $\mathrm{X}$ to prevent stenosis after rat carotid artery injury. Biomaterials. 2009;30:118-125. doi:10.1016/j.biomaterials.2008.09. 009

43. Prabaharan M. Chitosan-based nanoparticles for tumor-targeted drug delivery. Int J Biol Macromol. 2015;72:1313-1322. doi:10.1016/j. ijbiomac.2014.10.052

44. Morishima H, Washio J, Kitamura J, Shinohara Y, Takahashi T, Takahashi N. Real-time monitoring system for evaluating the acidproducing activity of oral squamous cell carcinoma cells at different environmental pH. Sci Rep-UK. 2017;7:10092. doi:10.1038/s41598017-10893-y
International Journal of Nanomedicine

\section{Publish your work in this journal}

The International Journal of Nanomedicine is an international, peerreviewed journal focusing on the application of nanotechnology in diagnostics, therapeutics, and drug delivery systems throughout the biomedical field. This journal is indexed on PubMed Central, MedLine, CAS, SciSearch ${ }^{\circledR}$, Current Contents ${ }^{\mathbb{R}} /$ Clinical Medicine,
Journal Citation Reports/Science Edition, EMBase, Scopus and the Elsevier Bibliographic databases. The manuscript management system is completely online and includes a very quick and fair peer-review system, which is all easy to use. Visit http://www.dovepress.com/ testimonials.php to read real quotes from published authors. 